\title{
Erratum to: Predicting Germination Probability of Radish (Raphanus sativus L.), Chinese Cabbage (Brassica rapa ssp. pekinensis), and Cabbage (B. oleracea var. capitata L.) Seeds via Amino Acid Leakage Parameters
}

\author{
Tai Gi Min ${ }^{1 *}$, Boseung Choi ${ }^{2}$, and Ba Reun Hong ${ }^{1}$ \\ ${ }^{I}$ Division of Life \& Environmental Science, College of Life \& Environmental Science, Daegu University, \\ Gyeongsan 712-714, Korea \\ ${ }^{2}$ Department of Computer Science \& Statistics, Daegu University, Gyeongsan 712-714, Korea \\ *Corresponding author: tgmin@daegu.ac.kr
}

(C) Korean Society for Horticultural Science and Springer 2013

Erratum to: Hort. Environ. Biotechnol. 54(5):388-398. 2013.

DOI 10.1007/s13580-013-0071-5

The original online version of this article contained an error in the Fig. 3.

The correct Fig. 3 is reproduced below. The print version was correct.
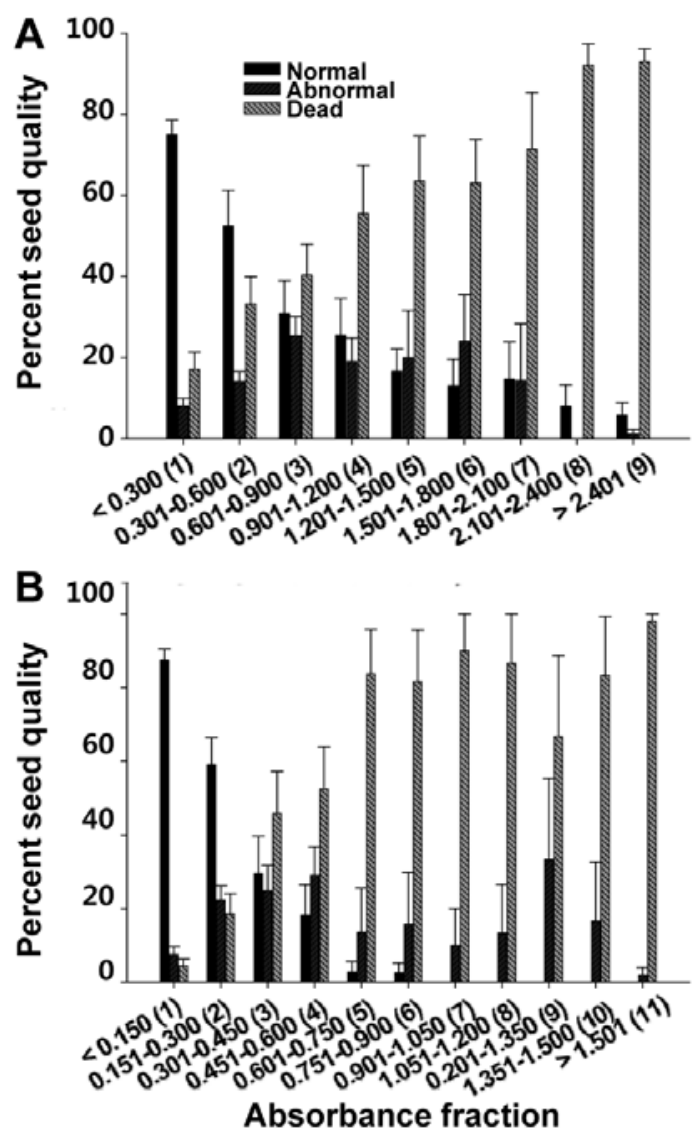

Fig. 3. Percent seed quality (normal, abnormal and dead seeds) of eight cultivars of radish $(A)$ and Chinese cabbage including one cultivar of cabbage (B) seeds depending on absorbance $(570 \mathrm{~nm})$ fractions of seed leachate with the NR treatment. Average of eight cultivars and vertical bars are standard errors.

The online version of the original article can be found under doi:10.1007/s13580-013-0071-5. 